$A D-A 059998$

UNCLASSIFIED

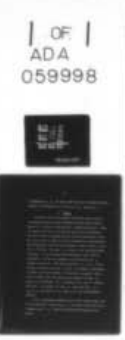

NORTH CAROLINA UNIV AT CHAPEL HILL DEPT OF STATISTICS

ESTIMATION IN THE GENERAL MULTIPLICATIVE MODEL FOR SURVIVAL. (U)

JUL $78 \mathrm{~N} L$ JOHNSON. R C ELANDT-JOHNSON MMS-1187

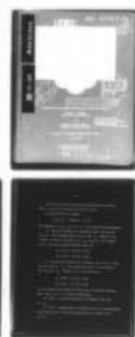

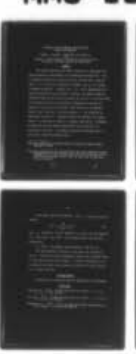

ARO-14778.3-M
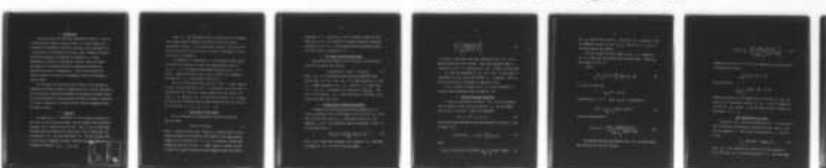

END

END
DATE

1278

DOC
F/G 12/1

DAAG29-77-C-0035

NL

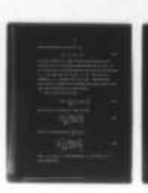




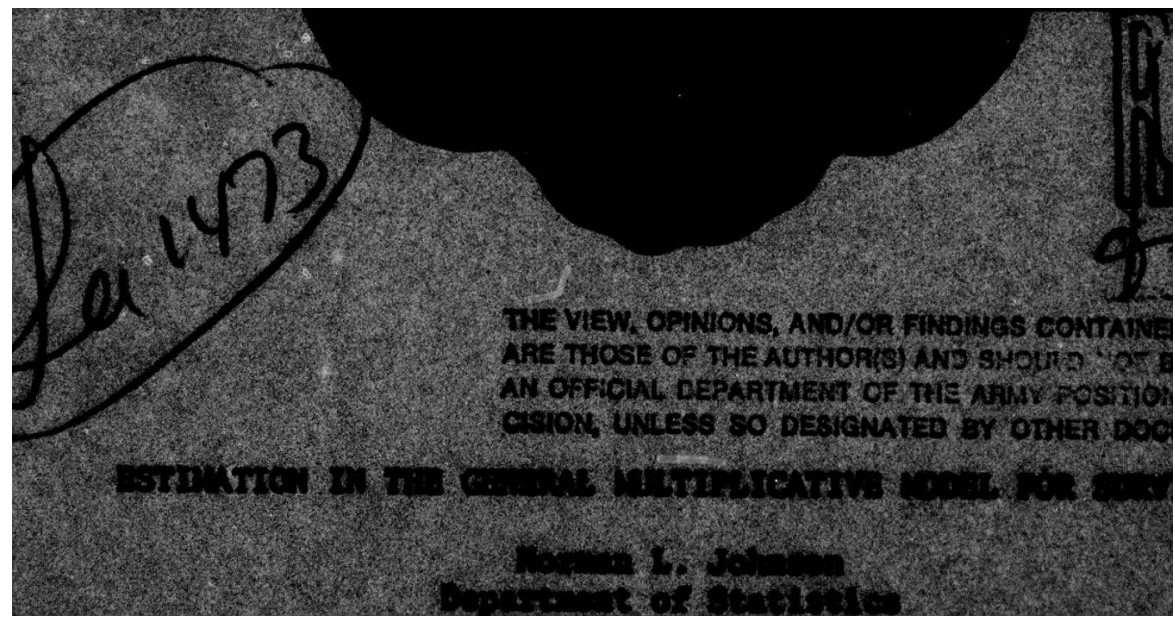




\title{
ESTIMATION IN THE GENERAL MULTIPLICATIVE MODEL FOR SURVIVAL
}

\author{
Norman L. Johnson*, Department of Statistics \\ and \\ Regina C. Elandt-Johnson**, Department of Biostatistics \\ University of North Carolina at Chapel Hill
}

\section{SUMMARY}

The general multiplicative model (formula (2)) represents the hazard function as the product of an "underlying" hazard rate, $\lambda(t)$, of unspecified form and a certain function of known form, $g(\underset{\sim}{z} ; \underset{\sim}{\beta})$, where $\underset{\sim}{ }$ is a vector of concomitant variables, and $\beta$ is a vector of unknown parameters. Assuming that $\lambda(t)$ can be approximated by a constant between any two consecutive failures, the general forms of likelihood function are derived (formulae (6) and (7), or (9) and (11)). The likelihood utilizes the available information on the time of exposure to risk of each individual (until failure or withdrawal). Special cases, when the $z$ 's do not depend on $t$ are discussed in some detail (Section 7). Multiple failures are handled in a simple manner - no ordering of failures is required (Section 8). Estimation of empirical survival function when there are no covariates is discussed in Section 9. An example using heart transplant data, is given (for illustrative purpose only).

\footnotetext{
* Research supported by the Army Office of Research, under Contract DAAG29-77-C-0035.

** Research supported by US. National Heart and Lung Institute Contract NIH-NHLI-71-2243 from the National Institutes of Health, and by Public Health Research Grant 1 R01 CA 17107 from the National Cancer Institute.
} 


\section{INTRODUCTION}

of recent years there has been considerable interest in the use of multiplicative models for hazard rates, as a means whereby the influence of concomitant variables on survival can be expressed, and so allowed for in the analysis of survival data. Methods of analysis have been developed for estimation of parameters, reflecting dependence of survival on concomitant variables, which are robust with respect to the form of hazard rate function (provided the multiplicative model is appropriate). Use of these methods does involve sacrifice of some of the information typically available in survival data.

In this paper the nature and likely effects of the omitted information are studied, mainly by comparison of the "critical functions" of the parameters reflecting dependence on concomitant variables which have to be maximized to obtain maximum likelihood estimators, according as to what information is included and what further assumptions about the model are made.

\section{NOTATION}

We suppose that $\mathrm{N}$ individuals in all are under observation at some time or other during the study. We also suppose that observation continues over a single interval of time - that is, that no individual withdraws and then reenters the study later. The i-thindividual will be denoted by (i); for each individual (i), the data include times of entry and of withdrawal or death (failure) and also-kalues-of s concomitant variables $\left(z_{1 i}, \ldots, z_{s i}\right)=z_{i}^{j} \cdot$

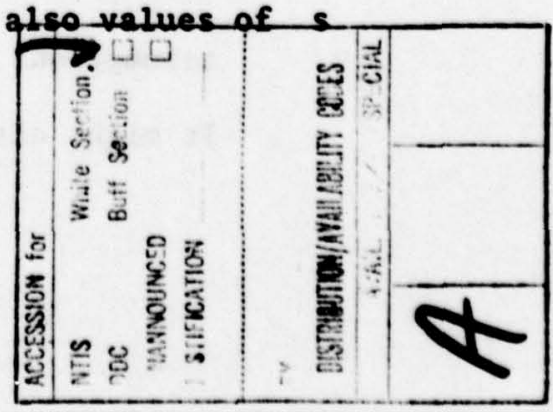


Time $t=0$ may correspond to date of initiation of a treatment (as in many clinical studies) or to date of birth (as in some occupational studies). In the first type of study it will very often be the case that the time of entry is 0 for all individuals, but this need not be so in general.

If, among the $\mathrm{N}$ individuals, $\mathrm{n}$ are observed to fail during the course of the study, we denote the ordered failure times by $t_{1}<t_{2}<\cdots<t_{n}$. We denote the individual failing at time $t_{j}$ by $(i(j))$. (Multiple failures, when $m_{j}(>1)$ failures are recorded at the same time, $t_{j}$, are discussed in Section 8.)

The set of individuals in the study at a time $t$ - those "exposed to risk" of being observed to fail - is called the risk set at time $t$. In particular the risk set at ("just before") the $j$-th failure - at time $t_{j}$ - is denoted by $R_{j}$. We also use the notation $R_{j}^{\prime}$ to denote the set of all individuals who are under observation for at least part of the interval $I_{j} \equiv\left(t_{j-1}, t_{j}\right] \quad(j=1, \ldots, n)$.

\section{FORMULATION OF THE PROBLEM}

We are interested in estimating the survival distribution function (SDF)

$$
S(t \mid \underset{\sim}{z})=\operatorname{Pr}[T<t \mid \underset{\sim}{z}]
$$

where $T$ denotes failure time. Usually it is impracticable to have sufficient numbers of individuals with common (or even approximately common) sets of values of the $z$ 's, so it is necessary to make some assumptions about the way the $z$ 's might combine to affect the SDF. It might also appear necessary to make assumptions about the form of 
dependence on $t$, specifying at least a parametric model for this. While this is so, it is possible to estimate parameters reflecting dependence on the $z^{\prime} s$, without making detailed assumptions about the form of SDF as a function of $t$.

\section{THE GENERAL MULTIPLICATIVE MODEL}

These methods are based on use of the general multiplicative model for the hazard rate function

$$
-d \log S(t \mid z \underset{\sim}{z}) / d t=\lambda(t \mid z \underset{\sim}{z})=\lambda(t) g(\underset{\sim}{z} ; \underset{\sim}{\beta}),
$$

where $\lambda(t)$ is an "underlying" hazard rate of unspecified form (except that $\lambda(t) \geq 0$ ), while $g(\cdot)$ is a known function of $\underset{\sim}{z}$ and of $r$ unknown parameters $\beta_{1}, \ldots, \beta_{r}$. Usually $r$ is taken equal to $s$, and $g(\cdot)$ is assumed to be a function of $\sum_{u=1}^{s} \beta_{u}^{2} u$, but this is not essential. If $\underset{\sim}{z}$ varies with time, we can write $\underset{\sim}{z}(t)$ on the right hand side of (2).

\section{MAXIMUM PARTIAL LIKELIHOOD ESTIMATORS}

A commonly used likelihood function for estimating the $\beta$ 's utilizes information only on individuals in the risk sets $R_{j}$. Given that a failure does occur at $t_{j}$, among the risk set $R_{j}$, and supposing $z=z(t)$, the probability that the individual $(i(j))$ is the one which fails is

$$
g\left(z_{i(j)}\left(t_{j}\right) ; \beta\right)\left[\sum_{\ell \in R_{j}} g\left(z_{\ell}\left(t_{j}\right) ; \beta\right)\right]^{-1},
$$

where $\ell \in R_{j}$ means that individual $(\ell)$ belongs to $R_{j}$. This does not depend on $\lambda(t)$. Cox (1972) used the product 


$$
\prod_{j=1}^{n}\left[\frac{g(\underset{\sim}{z}(j)}{\left.\sum_{\ell \in R_{j}} g\left(t_{j}\right) ; \beta\right)}\right] \text {, }
$$

as a "partial likelihood" from which estimation of the $B^{\prime} s$ can be found by maximizing this statistic. While this approach leads to estimators of the $\beta^{\prime}$ s uninfluenced by $\lambda(t)$ it should be noted that:

(i) Only the information $t_{j}, R_{j}$ and $(i(j))$ is used, that is, information on events inside the intervals $I_{j}$ is ignored. (This is, why the term "partial likelihood" is applied to (4));

(ii) In order to estimate $S(t \mid \underset{\sim}{2})$ it is still necessary to int roduce some assumptions about the form of $\lambda(t)$.

\section{MAXIMUM LIKELIHOOD ESTIMATION}

In order to approximate the form of $\lambda(t)$ it may be supposed that it remains constant over each interval, $I_{j}$, but can change from interval to interval. Under this assumption

$$
\lambda(t)=\lambda_{j} \text { for } t_{j-1}<t \leq t_{j} \text {, }
$$

and the likelihood function for the data described in Section 1, with the model (2) is

$$
L(\lambda ; \beta)=L\left(\lambda_{1}, \ldots, \lambda_{n} ; \underset{\sim}{\beta}\right)=\prod_{j=1}^{n} L_{j}\left(\lambda_{j} ; \underline{\beta}\right),
$$

where

$$
L_{j}\left(\lambda_{j} ; \underset{\sim}{\beta}\right)=\lambda_{j} g\left(z_{i(j)}\left(t_{j}\right) ; \underset{\sim}{\beta}\right) \exp \left[-\lambda_{j} \sum_{\ell \in R ! j} \int_{I_{j \ell}} g(\underset{\sim}{z}(t) ; \underline{\beta}) d t\right],
$$


and $I_{j} \ell$ denotes that part of $I_{j}$ over which $(\ell)$ is observed. Note the difference between $R_{j}^{\prime}$ and $R_{j}-R_{j}^{\prime}$ consists of $R_{j}$, plus all those individuals who withdraw.

We first maximize (6) with respect to the $\lambda_{j}$ 's (supposing the $\beta^{\prime} s$ fixed) and then maximize the resultant value, $L(\hat{\sim}(\beta) ; \beta)$, with respect to the $B^{\prime} s$.

Clearly

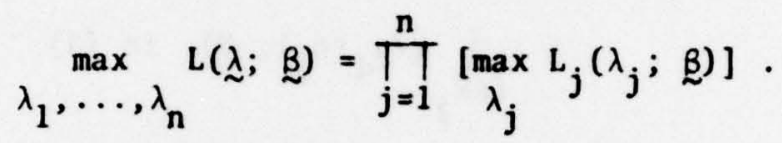

It is easy to show that

$$
\max _{\lambda} \lambda e^{-\lambda w}=w^{-1} e^{-1},
$$

corresponding to $\lambda=w^{-1}$. Hence $L(\lambda ; \beta)$ is maximized by

$$
\hat{\lambda}_{j}(\underset{\sim}{\beta})=\left[\sum_{\ell \in R ! j} \int_{I_{j \ell}} g(\underset{\sim}{z}(t) ; \underline{\sim}) d t\right]^{-1},
$$

and the maximized value is

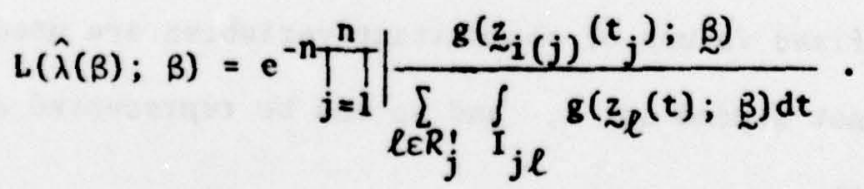

The maximum likelihood estimators of the $\beta^{\prime} s$ are those values which maximize the critical function 


$$
e^{n} L(\underset{\sim}{\hat{\lambda}(\beta) ; ~} \underset{\sim}{\beta})=\prod_{j=1}^{n}\left[\frac{g\left({\underset{\sim}{i}(j)}_{j}\left(t_{j}\right) ; \beta\right)}{\sum_{\ell \in R_{j}} \int_{I_{j \ell}} g\left({\underset{\sim}{z} \ell}^{(t ;} \underset{\sim}{\beta}\right) d t}\right] .
$$

Comparing (11) with (4) we see that the numerators are identical but the denominators differ,

$$
\sum_{\ell \in R_{j}} g\left({\underset{\sim}{z} \ell}_{j}\left(t_{j}\right) ; \beta\right) \text { in (4) }
$$

being replaced by

$$
\sum_{\ell \varepsilon R_{j}} \int_{I_{j \ell}} g(\underset{\sim}{z}(t) ; \underset{\sim}{\beta}) d t \text { in (11). }
$$

Whereas the quantity in (4) depends only on the risk set $\left(R_{j}\right)$ and values of the concomitant variables "at $t_{j}, "$ in (11) it depends on the risk set $\left(R_{j}^{\prime}\right)$ during $I_{j}$ and the whole periods of exposure of individuals $(\ell)$ in $I_{j}$.

\section{SOME IMPORTANT SPECIAL CASES}

In practice $\underset{\sim}{z}(t)$ will not be known for all $t$. In many cases, only fixed values of concomitant variables are used - that is $\underset{\sim}{z}(t)$ does not depend on $t$, and so can be represented as $\underset{\sim}{z}$. If this is so, then

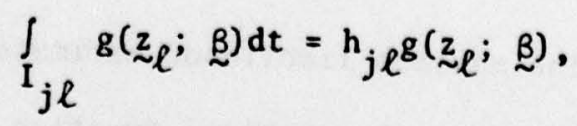

where $h_{j \ell}$ is the length of time ("person $\times$ time units exposed to risk") for which $(\ell)$ is under observation in $I_{j}$. For individuals 
under observation for the whole of $I_{j}$,

$$
h_{j \ell}=t_{j}-t_{j-1}=h_{j} \text {. }
$$

If precise information on times of entry and withdrawal are not available, more or less arbitrary approximations must be used. If

( $\ell$ ) either enters or withdraws from the study (but does not do both) in $I_{j}$ one might take $h_{j \ell} \div \frac{1}{2} h_{j}$; if $(\ell)$ both enters and withdraws in $I_{j}$, one might take $h_{j \ell} \div \frac{1}{3} h_{j}$. Sometimes there may be specific information on withdrawal and/or entry "habits" which might lead to modification of these formulae.

From (9) and (12) we see that

$$
\hat{\lambda}_{j}(\beta)=\left[\sum_{\ell \in R !} h_{j \ell} g(\underset{\sim}{z} ; \underset{\sim}{\beta})\right]^{-1},
$$

and the critical function (11) takes the form

$$
\prod_{j=1}^{n}\left[\frac{g(\underset{\sim}{z}(j) ; \beta)}{\sum_{\ell \in R_{j}} h_{j \ell} g\left(\underset{\sim}{z} \ell^{\prime} ; \beta\right)}\right] \text {. }
$$

This is a constant multiple $\left(\prod_{j=1}^{n} h_{j}^{-1}\right)$ of

$$
\prod_{j=1}^{n}\left[\frac{g\left(z_{i}^{z}(j) ; \underline{\alpha}\right)}{\sum_{\ell \in R !} \theta_{j \ell} g(\underset{\sim}{z} ; \underline{\alpha})}\right] \text {, }
$$

where $\theta_{j \ell}=h_{j \ell} / h_{j}$ is the proportion of $I_{j}$ for which $(\ell)$ is under observation. 
The critical function (partial likelihood) (4) is now

$$
\prod_{j=1}^{n}\left[\frac{g\left(z_{i}(j) ; \beta\right)}{\sum_{\ell \in R_{j}} g(\underset{\sim \ell}{z} ; \beta)}\right]
$$

Comparison of (16) and (17) shows that they differ in their denominators: - (a) the summation in (16) is over $R_{j}^{\prime}$, while that in $\left(17\right.$, is over $R_{j}$, thus omitting individuals withdrawing in $I_{j}$; (b) the factors $\theta_{j \ell}$ in (16) reflect different proportions of $I_{j}$ during which the corresponding individuals were under observations. To sum up, (17) could be obtained from (16) by (a) ignoring individuals who withdrew during $I_{j}$, and (b) supposing all individuals who enter (and do not withdraw) during $I_{j}$ to have been under observation for the whole of the interval.

Situations when there are no new entries deserve, perhaps, special attention. These are typical in clinical trials, where $t$ is the follow up time since initiation of the treatment.

For computational purposes, it might be useful to represent (16) and (17) in more convenient forms.

Let $\tau_{\ell}$ denote the time of departure (by failure or withdrawal) for individual $(\ell)$.

Then

$$
\theta_{j \ell}= \begin{cases}0 & \text { for } \tau_{\ell} \leq t_{j-1} \\ \left(\tau_{\ell}-t_{j-1}\right) / h_{j} & \text { for } t_{j-1}<\tau_{\ell}<\tau_{j} \\ 1 & \text { for } \tau_{l} \geq t_{j},\end{cases}
$$


so that (16) takes the form

$$
\prod_{j=1}^{n}\left[\frac{g(\underset{\sim i(j)}{z} ; \underset{\sim}{\beta})}{\sum_{\ell=1}^{N} \theta_{j \ell} g(\underset{\sim}{z} ; \beta)}\right] .
$$

If we were to define

$$
\theta_{j \ell}=\left\{\begin{array}{lll}
0 & \text { if } & \tau>t_{j} \\
1 & \text { if } & \tau \geq t_{j},
\end{array}\right.
$$

formula (19) would correspond to (17).

\section{MULTIPLE FAILURES}

If several failures $-m_{j}$, say - are recorded at the same time, then, in practice, it means that the time unit of record is not sufficiently small to distinguish them. However, it is possible to establish a likelihood function, treating the failures as if they really did occur at the same time, and so to obtain maximum likelihood estimators.

We denote the $m_{j}$ individuals failing at time $t_{j}$ by $(i(j, 1)),(i(j, 2)), \ldots,\left(i\left(j, m_{j}\right)\right)(j=1,2, \ldots, n)$. of course, we must have $m_{1}+m_{2}+\ldots+m_{n} \leq N$.

The likelihood function is

$$
L(\lambda ; \beta)=\prod_{n=1}^{n}\left[\lambda_{j}^{m}\left\{\prod_{k=1}^{m} g\left(\underset{\sim}{z_{j}}(j, k)\left(t_{j}\right) ; \beta\right)\right\} \exp \left\{-\lambda_{j} \sum_{\ell \in R_{j}^{\prime}} \int_{I_{j \ell}} g\left(\underset{\sim}{z} \ell^{(t)} ; \beta\right) d t\right]\right\} .
$$


Maximizing with respect to $\lambda$ gives

$$
\hat{\lambda}_{j}(\underline{\beta})=m_{j}\left[\sum_{\ell \in R_{j}^{\prime}} \int_{I_{j \ell}} g\left({\underset{\sim}{z}}_{\ell}(t) ; \underset{\sim}{\beta}\right) d t\right]^{-1},
$$

and the maximized value of the likelihood is

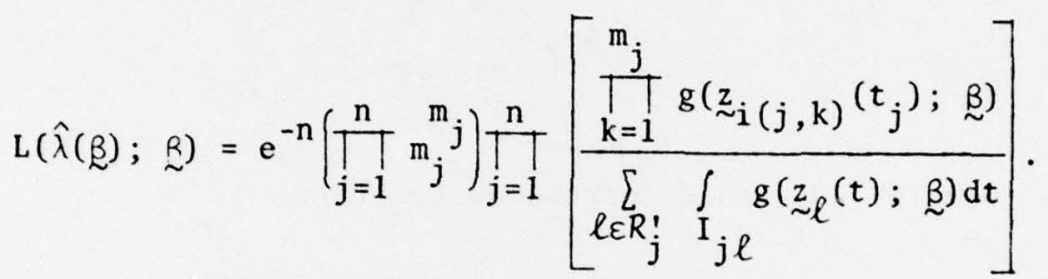

If $I_{j \ell} \equiv I_{j}$ in (22), we cbtain Breslow's (1972) formula.

The $\beta^{\prime} s$ are obtained by maximizing the critical function

$$
e^{n}\left(\prod_{j=1}^{n} m_{j}^{m_{j}}\right)-1 L(\underset{\sim}{\hat{\lambda}}(\beta) ; \beta)=\prod_{j=1}^{n}\left[\frac{\prod_{k=1}^{m_{j}} g\left(\underset{\sim}{z} i(j, k)\left(t_{j}\right) ; \underset{\sim}{\beta}\right)}{\sum_{\ell \in R_{j}} \int_{I_{j \ell}} g(\underset{\sim}{z}(t) ; \beta) d t}\right],
$$

which differs from (11) only by substitution of

$$
\prod_{k=1}^{m_{j}} g\left(\underset{\sim i(j, k)}{z}\left(t_{j}\right) ; \underset{\sim}{\beta}\right) \text { for } g\left({\underset{\sim}{z}}_{i(j)}\left(t_{j}\right) ; \underset{\sim}{\beta}\right),
$$

in the numerator.

If the $z$ 's do not depend on $t$, then in place of (24) we can use the critical function

$$
\prod_{j=1}^{n}\left[\frac{\prod_{k=1}^{m_{j}} g(\underset{\sim i}{z}(j, k) ; \beta)}{\sum_{\ell R_{j}} \theta_{j \ell} g(\underset{\sim}{z} ; \beta)}\right]
$$


9. ESTIMATION OF SURVIVAL FUNCTION WHEN THERE ARE NO COVARIATES

When the covariates are absent so that $g(\cdot) \equiv 1$, formula (22) gives

$$
\hat{\lambda}_{j}=m_{j}\left\{\sum_{\ell \in R ! j} h_{j \ell}\right\}^{-1} .
$$

The corresponding estimate of the SDF (for $t_{j-1} \leq t<t_{j}$ ) is

$$
\begin{aligned}
s \hat{(t)} & =\exp \left\{-\sum_{f=1}^{j-1} \hat{\lambda}_{f} h_{f}-\hat{\lambda}_{j}\left(t-t_{j-1}\right)\right\} \\
& =\exp \left[-\sum_{f=1}^{j-1} m_{f}\left(\sum_{\ell \in R_{f}^{\prime}} \theta_{f l}\right)^{-1}-m_{j}\left(t-t_{j-1}\right) h_{j}^{-1}\left(\sum_{\ell \varepsilon R_{j}^{\prime}} \theta_{j \ell}\right)\right] \\
& \text { for } t_{j-1} \leq t<t \quad(j=1, \ldots, n) .
\end{aligned}
$$

For $t>t_{n}$ we have $\left.\hat{s(t)}=s \hat{(t}_{n}\right)$ formally, but there may be relatively few data for these $t$ (they correspond to individuals under observation for longer periods than that to the last failure).

If $\theta_{f \ell} \equiv 1$ in (16) - that is, there are no new entries, and if $R_{f}^{\prime} \equiv R_{f}$ - that is, there are no withdrawals - then (27) gives

$$
\hat{S(t)}=\exp \left[-\sum_{f=1}^{j-1} m_{f} R_{f}^{-1}-m_{j} R_{j}^{-1}\left(t-t_{j-1}\right) h_{j}^{-1}\right], t_{j-1} \leq t<t{ }_{j} .
$$

By comparison, the Kaplan-Meier estimator of $S(t)$ is

$$
\hat{S}^{\star}(t)=\prod_{f=1}^{j-1}\left(1-m R_{f}^{-1}\right) \quad\left(t_{j-1} \leq t<t_{j}\right),
$$

where $R_{f}$ is the number of individuals in $R_{f}$.

Another approximation, given by Thompson (1977), leads to a formula like (29), but with $R_{f}$ increased by one-half of the number 
of withdrawals in $I_{f}$. Of course, when there are no multiple failures, formulae (22) through (29) are valid with $\mathrm{m}_{\mathrm{f}}$ 's replaced by 1 .

\section{EXAMPLE}

The data in Table 1 are adopted from Crowley and Hsu (1974) ("Covariance Analysis of Heart Transplant Survival Data," Technical Report No. 2, Division of Biostatistics, Stanford University). These are survival data for $\mathrm{N}=64$ patients who had heart transplants. The date of entry corresponds here to the date of operation, so that the time at entry is equal to zero for each patient in the follow-up. The investigation covers the period January 6, 1968 to April 1, 1974 , that is 2276 days. The time $\tau_{\ell}$ (in days) is the time beyond which individual ( $\ell$ ) was no longer under observation (after death or withdrawal). The data are arranged in increasing order of ${ }^{\tau} \ell$ (column 3 of Table 1). Note that for patient (1) $\tau_{1}=0^{+}$; we have assigned arbitrarily the value 0.5 days. The numbers in parentheses in column 1 are the ID numbers of the patients, assigned to them at entry. Column 2 gives the values of the indicator variable $\delta_{\ell}$ ' equal to 1 if individual $(\ell)$ is observed to die, and 0 otherwise. There were $n=39$ deaths, at times $t_{j}$. (Note that when $\delta_{\ell}=1$, $\left.{ }^{\top} \ell(j)=t_{j}.\right)$ The values of $\tau_{\ell}$, when $\delta_{\ell}=0$ are distinguished by asterisk.

Out of 8 concomitant variables given in the original paper, only two are shown here: age at entry, $z_{1}$ (in years), and the so called "mismatch score", $z_{2}$, based on tissue and blood typing of each patient . 
We use these data for illustrating the methods of estimation. There is no claim to evaluate the study as a whole.

The model used here is simply

$$
\lambda(\underset{\sim j}{t} ; \underset{\sim j}{z})=\lambda_{j} \exp \left(\beta_{1} z_{1 j}+\beta_{2} z_{2 j}\right) .
$$

The parameters $\beta_{1}, \beta_{2}$, and the $\lambda_{j}$ 's are estimated by two approaches.

(a) Using the information on withdrawal times, with the risk sets $R_{j}^{\prime}$. The likelihood function (19), with $\theta_{j l}$ defined in (18), is used. We notice a multiplicity at $t_{17} \quad\left(m_{17}=2\right)$. The estimates of $\beta^{\prime}$ 's and their estimated standard errors are:

$$
\begin{aligned}
& \hat{\beta}_{1}=0.0705, \quad \text { S.D. }\left(\hat{\beta}_{1}\right)=0.0233 ; \\
& \hat{\beta}_{2}=0.6644, \quad \text { S.D. }\left(\hat{\beta}_{2}\right)=0.2876 .
\end{aligned}
$$

(b) Ignoring che information on withdrawal time, that is, using only the risk sets, $R_{j}$. The likelihood function is still given by (19), but with $\theta_{j \ell}$ defined by (20). The results are:

$$
\begin{aligned}
& \hat{\beta}_{1}=0.0699, \quad \text { S.D. }\left(\hat{\beta}_{1}\right)=0.0234 \\
& \hat{\beta}_{2}=0.6817, \quad \text { S.D. }\left(\hat{\beta}_{2}\right)=0.2883 .
\end{aligned}
$$

We also evaluate the estimated survival functions ignoring covariates, that is when $g(\cdot)=1$, in three different ways.

(a) $\hat{S} 1(t)$ - utilizing information on withdrawal times with sets $R_{j}^{\prime}$;

(b) $\hat{\mathrm{S} 2}(\mathrm{t})$ - assuming that the withdrawal time is at the beginning of the interval for all those who withdraw in the interval, with sets $R_{j}$. 
Since there were no new entries, $\lambda_{j}(t)$, is estimated from the formula

$$
\hat{\lambda}_{j}(t)=m_{j}\left[\sum_{\ell=1}^{64} \theta_{j \ell} h_{j}\right]^{-1},
$$

with $\theta_{j \ell}$ defined by (18) for appraoch (a), and by (20) for approach (b). Then $\hat{S} 1(t)$ and $\widehat{S 2}(t)$ are calculated from (27) and (28), respectively.

(c) $\widehat{S^{\star}}(t)$ - the product-limit estimates, with sets $R_{j}$.

The resulting SDF's are given in the three last columns of

Table 1. There are not many withdrawals between any two deaths (this is especially true for early deaths). In these circumstances is to be expected that these three estimates of SDF do not differ greatly as is, indeed, the case.

\section{ACKNOWLEDGEMENT}

We would like to thank Paul Wan for computation in the Example.

\section{REFERENCES}

Breslow, N.E. (1972). Discussion of D.R. Cox (1972) J.R. Statist. Soc. Ser. B $33,216-217$.

Cox, D.R. (1972). Regression models and life tables. J.R. Statist. Soc. Ser. B 33, 187-202.

Thompson, W.A. (1977). On the treatment of grouped observations in life studies. Biometrics $\underset{\sim}{33}, 463-470$. 
TABLE 1

Estiation of Survival Function for lleart Transplant Data

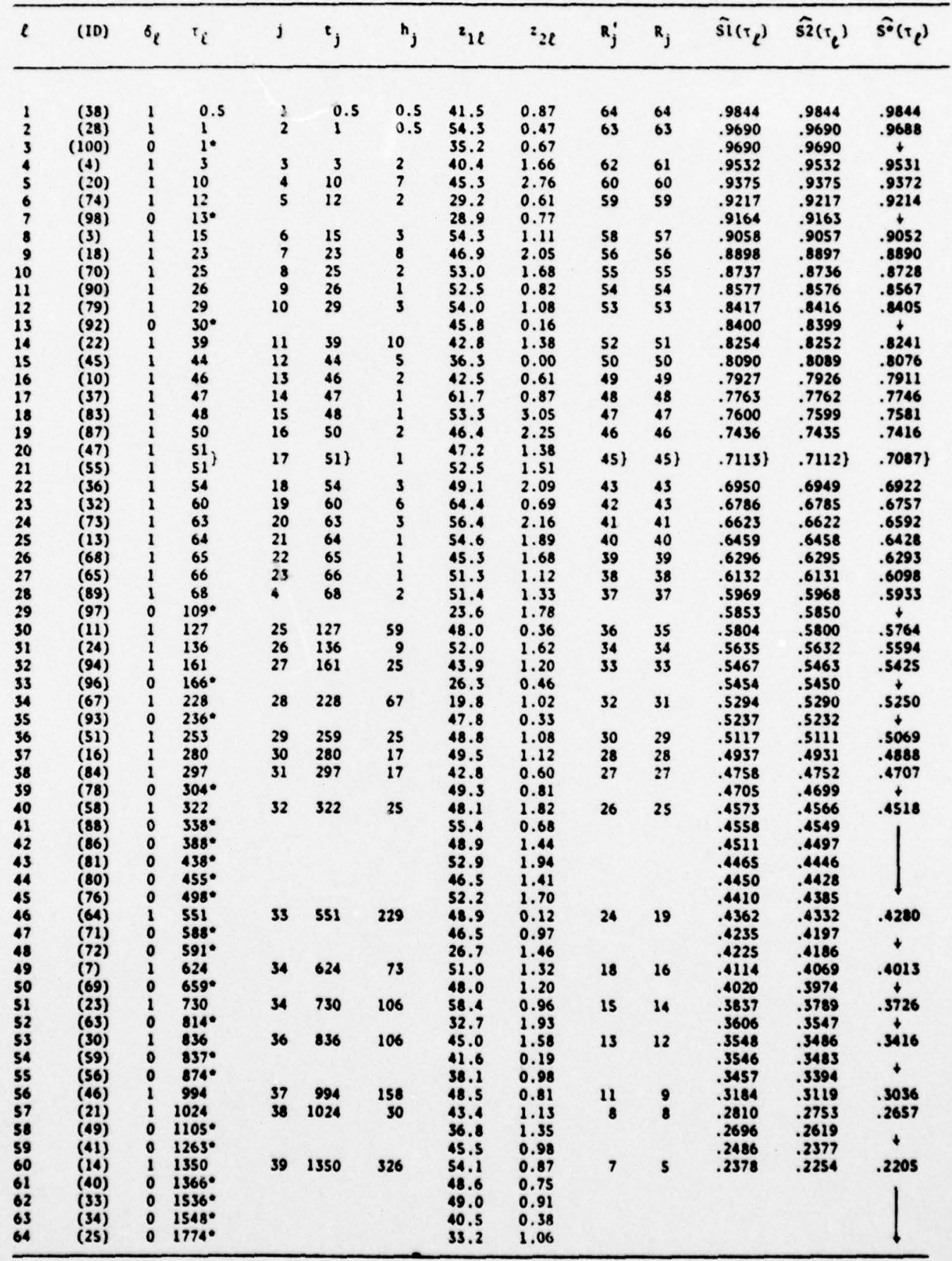


UNCLASSIFIED

SECURITY CLASSIFICATION OF THIS PAGE (Whon DOTOEnIOTOd),

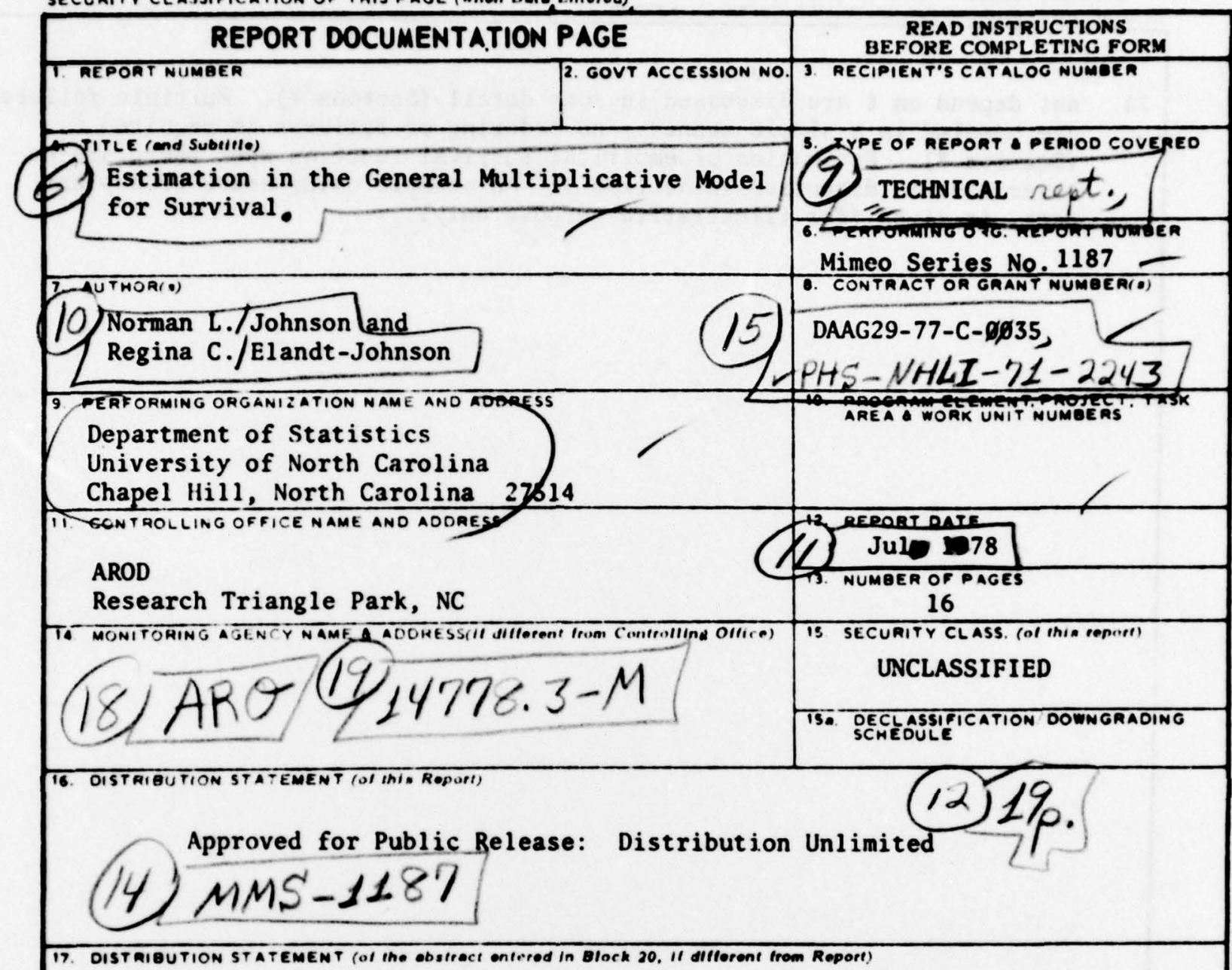

10. SUPPLEMENTARY NOTES

19. KEY wOAOS (Continue on reveree side if nocesecery and Identily by block number)

Estimation, Multiplicative mortality model, Maximum likelihood, Partial likelihood, Ties, Multiple deaths

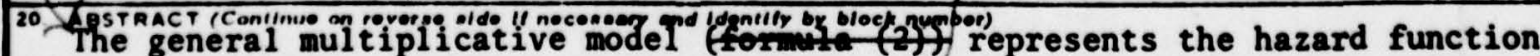
as the product of an "underlying hazard rate, $x(t)$, of unspecified form and a certain function of known form, $g(z ; \beta)$, where $z$ is a vector of concomitant variables, and $B$ is a vector of unknowin parametẽrs. Assuming that $\chi(t)$ can be a mm approximated by a constant between any two consecutive failures, the general forms of likelihood function are derived, (formutec $(6)$ and $(7)$, or $(9)$ and (11)). The likelihood utilizes the available information on the time of exposure to risk of each individual (until failure or withdrawal). Special cases, when the $z$ 's do 


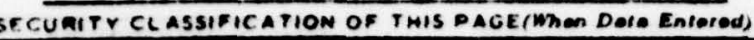

r

20. not depend on $t$ are discussed in some detail, fsection-7t Multiple failures are handled in a simple manner - no ordering of failures is required. (Eection 8). Estimation of empirical survival function when there are no covariates is discussed in-section 9 . An example using heart transplant data, is given (for illustrative purpose only). 